\title{
Treatment Planning of Cancer Dendritic Cell Therapy Using Multi-Objective Optimization
}

\author{
K. Lakshmi Kiran*, S. Lakshminarayanan* \\ *Department of Chemical and Biomolecular Engineering, National University of Singapore, 117576 \\ Singapore (Tel: 0065-65168484; e-mail: chels@nus.edu.sg).
}

\begin{abstract}
Cancer immunotherapy is one of the emerging therapies for cancer treatment where immune cells are guided to fight against cancer. Clinical immunologists are proposing different ideas to stimulate the immune cells and dendritic cell therapy is one among them. Like, other treatment modalities, the challenge in dendritic cell therapy is when and how much dendritic cells should be administered. In this work we use a mathematical model which elucidates the activation of the helper T-cells and cytotoxic Tcells by the intervention of dendritic cells. The objective is to minimize the tumor cells for a given input of dendritic cells. Then multi-objective optimization is applied on the model to design the treatment planning in order to achieve the objective.
\end{abstract}

Keywords: Cancer, Immune system, Dendritic cell therapy, Mathematical model, Multi-objective optimization

\section{INTRODUCTION}

Cancer stands next only to heart disease in the list of most fatal diseases in the world. From Fig.1, it is obvious that the decrease in death rate for cancer patients over the years 19502003 has been minimal as compared to other major diseases. Cancer related deaths have been escalating meteorically according to World Health Organization, 7.6 million people died of cancer (out of 58 million deaths overall) in 2005 . They speculate that cancer deaths will increase to $18 \%$ and $50 \%$ by 2015 and 2030 respectively. Recently, the American Cancer Society reported that around 1.5 million new cancer cases and 0.6 million cancer death cases occurred in the US in 2007. According to another report on worldwide cancer rates by the WHO's International Agency for Research on Cancer (IARC) (Paola Pisani, 2002), North America leads the world in the rate of cancers diagnosed in adults, followed closely by Western Europe, Australia and New Zealand. In 1994, in Britain, almost one in three were expected to develop the disease over their survival period and it is estimated to increase to one in two by 2010 with reference to the trends at that time (Imperial Cancer Research Fund). Another publication from the Australian Institute of Health and Welfare (1999) projects that, based on the incidence rates existing in 1999, one in three men and one in four women would be directly affected by cancer in the first 75 years of life. Moreover, a loss of 254,000 potential years of life to the community each year was estimated as a result of people dying of cancer before the age of 75 . As a whole, cancer is currently responsible for $29 \%$ of male deaths and $25 \%$ of female deaths in Australia. In Singapore, the proportion of cancer deaths among all causes of death rose steadily from $14.8 \%$ (in the years $1968-1972$ ) to $27.1 \%$ (in the period 1998 2002) reflecting a worldwide trend. The above mentioned figures are alarming and have drawn the attention of researchers to understand the mechanism of cancer and come out with better therapies.

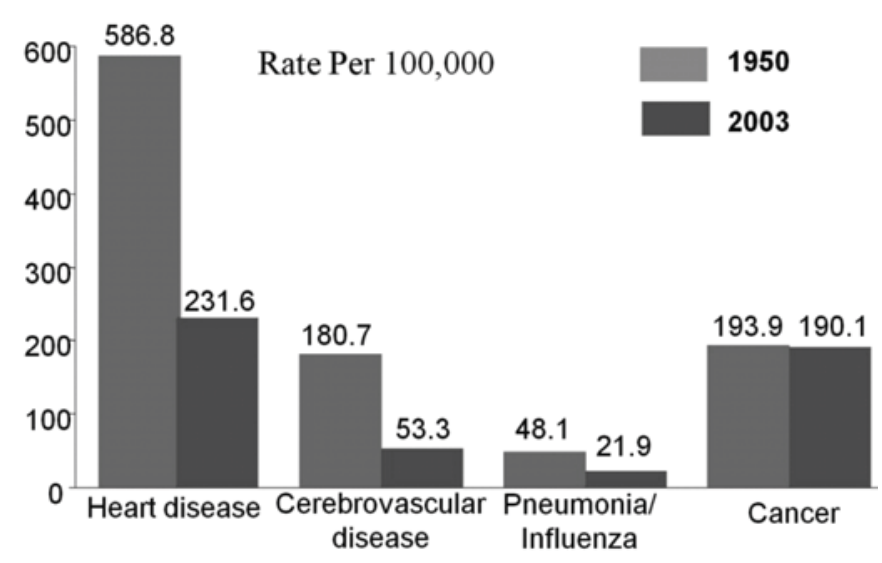

Fig.1. Change in death rates of different diseases in US from 1950 to 2003

The main characteristics of the cancer cell are its uncontrolled and unregulated growth (Hanahan and Weinberg, 2000; Martins et al., 2007). It is caused by the external factors such as UV radiations, carcinogenic chemicals as well as transfer of the cancer prone genes from parents. When a normal cell interacts with these external factors, its information system (DNA) gets damaged and the normal cell transforms to a cancer cell. Initially, the clump of the cancer cells is confined to particular location and it is regarded as being benign. If the cancer is not diagnosed and treated in the benign stage, it will change into a malignant form, and the cancer cells could migrate to different parts of the body and ultimately may lead to the death of the patient. So, it is better for the cancer patients to be provided suitable 
treatment at the early stages itself so as to prolong and enhance the quality of their life.

\subsection{Cancer treatment modalities}

Over the past 50 years, many cancer treatment modalities have been discovered. The most prominent of these are surgery, chemotherapy, and radiation therapy. Some of the emerging therapies are immunotherapy and viral therapy. However, a specific therapy for all types of cancer is still missing and the available therapies have their own advantages and shortcomings. Surgical techniques to remove tumors have been in practice even thousand years ago. Usually, surgery is favoured to remove the tumors diagnosed at the very early stages ensuring almost complete cure. Surgery can be very difficult if the tumor location is near critical locations in the body (e.g. brain); furthermore, surgery is not a preferred option if the cancer had already metastasized by the time of diagnosis. In any case, complete clearance of the tumor cells is not assured with surgery. Radiation therapy is an alternative to surgery in order to kill the localized tumor cells. In radiation therapy, high energy radiations are used and its dosage is determined such that the normal cells near the tumor are spared. Thus, radiation therapy also depends on the location of the tumor and sensitivity of the tumor cells to the radiation.

As a result, surgery is followed by chemotherapy or radiation therapy to suppress further tumor growth. In the case of chemotherapy and radiation therapy, precise care should be taken to avert the damage to normal tissues. Among these two therapies, chemotherapy is preferred because it is a systemic therapy. In systemic therapy, the drug flows throughout the body and destroys the migrated cancer cells along with residual cancer cells near the surgery location. Chemotherapy is always given as a course in cycles based on the patient health status rather than as a one-time treatment. This is done so as to maintain the drug concentration within the dosage limits in the body and kill the remaining cancer cells in the subsequent treatments (Dua et al., 2008). However, the side effects of the chemotherapy are significant and sometimes they become serious than the disease itself. In contrast to chemotherapy and radiation therapy, immunotherapy has fewer side effects, because, typically, the patient's own cells are modified and used as therapeutic agents. The prime objectives of any therapy are to keep the number of cancer cells below a lethal level and avoid the side effects caused by the therapeutic agents. This can be achieved by optimal scheduling and the optimal administration of the therapeutic agents if the dynamical characteristics of the system are known.

\section{CANCER IMMUNOTHERAPY}

The role of the immune system in cancer treatment was first observed by William Coley, a New York surgeon. Cancer immunotherapy is the stimulation of immune cells to fight the tumor cells. The main function of the immune system is to fight against the abnormal changes in the body, and the successful functioning of it lies in its ability to distinguish the "self" and "non-self" based on the self-marking molecules. The immune system recognizes the abnormality with the help of antigens presented by the injured or abnormal cells. If the immune system exhibits a response based on antigen recognition, then antigens are called immunogenic. However, not all antigens are immunogenic. For example, if the tumor is larger in size, immune cells may not respond to it. Before studying the tumor-immune interactions, it is informative to take a brief look at the mechanisms of the immune system. In this regard, the review paper by Adam et al., (2003) provides a comprehensive discussion on the immune response in cancer.

In our body, the immune action is carried out by specialized cells called lymphocytes which are mostly present in the blood. The common lymphocytes are macrophages, dendritic cells, natural killer (NK) cells, lymphokine activated killer cells, B-cells and T-cells. Immune response is categorized as natural immunity, humoral immunity and cellular immunity based on the lymphocytes. Macrophages, dendritic cells, and natural killer cells are responsible for natural immunity, in which these cells directly attack the infected cells (cancer cell) and act as antigen presenting cells (APC). Antigen is an agent which can easily be recognized by immune cells. Thus, APC highlights the infected cells and alerts the T-cells for further action against the infected cells. In humoral immunity, antibodies produced by B-cells encounters the infected cells. Each B-cell has a specific antibody of a particular shape. The concept of antibody-antigen interaction resembles the mechanism of lock and key. When the shape of an antibody of a B-cell matches exactly with the shape of the antigen corresponding to the infected cells, B-cell proliferates and produces plasma cells which actively secrete the antibodies. These antibodies neutralize the activity of the infected cell by inhibiting their cell division process, by producing a lethal group of enzymes called complement and by opsonization. In opsonization, antibodies coat the infected cells in order to make them easily recognizable by the killer lymphocytes. This process is known as antibody dependent cell-mediated cytotoxicity. In cellular immunity, the key players are T-cells which are further classified as helper Tcells $\left(\mathrm{CD}^{+}\right)$and cytotoxic T-cells $\left(\mathrm{CD}^{+}\right)$. Helper T-cells gets activated by the natural immune cells and regulates the production of the cytokines. Cytokines are the enzymes which keep the momentum of all the immune cells as per their requirement. Interleukins and interferons are regarded as the important cytokines to fulfil the immune action. Cytotoxic T-cells directly attack the infected cells after its activation by the cytokines.

From the above discussion, we can infer that key elements for immunotherapy are antibodies, cytokines, and the natural immune cells. On this basis, immunotherapy is classified into three main schemes, monoclonal antibody therapy (MAT), adoptive cellular immunotherapy (ACI) and vaccines (Adam et al., 2003). MAT involves the introduction of externally developed tumor specific antibodies into the patient's body using hybridoma technology. ACI constitutes the 
modification of the lymphocytes (helper T-cells, cytotoxic Tcells, NK cells) by using cytokines like interleukin (Kirschner and Panetta, 1998). This ultimately ameliorates the antitumor activity of the lymphocytes. This is done in two ways namely, lymphokine-activated killer cell (LAK) therapy and tumor infiltrating lymphocyte (TIL) therapy. In both therapies, the lymphocytes are activated externally and later they are injected back at the tumor site. In LAK therapy, the lymphocytes are obtained from the in vitro culturing with high concentration of IL-2 which is extracted from the patient's blood. Conversely, in TIL therapy, lymphocytes are taken from the patient tumor sites and cultured with the high concentrations of interleukin in vitro.

Generally, cancer vaccines are in the form of cells, molecules or micro-organisms. They facilitate the quick recognition of tumor cells by the immune cells. In other words, tumor associated antigens (TAA) are made tangible to the immune cells. Consequently, tumor-immune interactions are enhanced thereby achieving the goal of cancer immunotherapy.

\section{TUMOR-IMMUNE INTERACTION MODELS}

Tumor-immune interaction models explain interactions between different types of immune cells and the tumor. There are many tumor-immune interaction models and a few of them will now be described. In the model proposed by De Boer and Hogeweg (1986), interactions between macrophages, T-lymphocytes and tumors are considered. The macrophages and T-lymphocytes are given in different dosages. This model captures the "sneaking through" phenomenon i.e. when lower dosages of tumor is introduced, the immune system may not recognize it and then the tumor grows to a bigger size; however, the immune cells reject the tumor when they are given in higher quantities. Another successful model (Kuznetsov et al., 1994) was developed based on the studies of the B-lymphoma $\mathrm{BCL}_{1}$ in the spleen of mice. The model is very simple, and considers only two states (effector and tumor cells). Effector cells represent any of the killer immune cells. In this model, the parameters were estimated using in vivo data. Then, bifurcation analysis was performed to find the critical parameters for sneak through phenomenon. This model was further extended and modified (Kirschner and Panetta, 1998) by including the dynamics of the interleukin. Based on bifurcation analysis, this work emphasized that tumor immunogenicity is an important parameter. Their work also discussed about the effects of immunotherapy (adoptive cellular therapy and interleukin) through mathematical analysis. Models such as those by (Castiglione and Piccoli, 2007; de Pillis et al., 2005; de Pillis et al., 2006), consider the natural killer cells and cytotoxic $\mathrm{CD}^{+} \mathrm{T}$ cells as different states rather than considering them under the same family as effector cells. In de Pillis et al. (2005), the authors focus on the impact of NK cells and $\mathrm{CD} 8^{+} \mathrm{T}$ cells on tumor growth. Their model is in the form of a system of ODEs. Parameters of the model were estimated and validated with the published mice and human data. In addition, sensitivity analysis was done on the model. The sensitivity analysis concluded that the variable to which model is sensitive is patient-specific. This model was extended with slight modifications in the functional forms of growth and death terms of the immune cells (de Pillis et al., 2006). The model was also used for understanding the effects of combination therapy (chemo-immuno and vaccine therapy) for different patient parameters. Other models have included the dynamics of NK cells, B cells, helper and cytotoxic T-cells , and LAK cells (Szymanska, 2003). In this work, dendritic cell vaccine (DCV) is considered. DCV is produced by the process called dendritic cell transfection. In this process, some TAA are configured and cultivated with autologous dendritic cells that is extracted from the patient itself (Cappuccio et al., 2007; Castiglione and Piccoli, 2007; Piccoli and Castiglione, 2006). The resulting vaccine is injected back into the patient. Here, we consider the mathematical model proposed by Piccoli and Castiglione (2006) because this is the only model which includes the dynamics of dendritic cells and apply multi-objective optimization using non-dominated sorting genetic algorithm (NSGA) to find the optimal scheduling of dendritic cell vaccine interventions.

\section{MULTI-OBJECTIVE OPTIMIZATION}

Multi-objective optimization (MOP) is the optimization of two or more conflicting objectives of a system represented in the form of a mathematical model subjected to certain known constraints. Most practical problems such as product and process design, finance, aircraft design, automobile design, and medical applications have multiple objective scenarios. In these problems, an optimal decision needs to be taken in the presence of trade-offs between the conflicting objectives. In MOP, there may be a number of solutions in the feasible region, and the decision maker has to analyze all the solutions based on the prior knowledge of the system before a final solution is adopted (Tamaki et al., 1996).

Suppose there are ' $\mathrm{n}$ ' decision variables and 'p' objectives. MOP tries to find a point $x=\left(x_{1}, \ldots \ldots \ldots, x_{n}\right)$ which minimizes (or maximizes) the values of the objective functions $f=$ $\left(f_{1}, \ldots \ldots, f_{p}\right)$ within the feasible region $F$ of $x$. In contrast to single-objective optimization problems, an exact solution may not exist for the MOP problems because of the trade-off characteristics among the objectives. Hence a concept of the Pareto-optimal set was introduced for MOP problems. Pareto-optimal set is, 'a family of points which is optimal in the sense that no improvement can be achieved in any objective without degradation in others'.

Definition (Tamaki et al., 1996): Let $x^{0}, x^{1}, x^{2} \in F$

1. $x^{1}$ is said to be dominated by (or inferior to) $x^{2}$, if $f\left(x^{1}\right)$ is partially less than $f\left(x^{2}\right)$, i.e.,

$f_{i}\left(x^{1}\right) \geq f_{i}\left(x^{2}\right), \forall_{i}=1, \ldots \ldots, p$, and $f_{i}\left(x^{1}\right)>f_{i}\left(x^{2}\right), \exists_{i=1, \ldots \ldots .}$. 
2. $x^{0}$ is the Pareto-optimal (or non-dominated), if there doesn't exist any $x \in F$ such that $x$ dominates $x^{0}$

As Pareto-optimal solution is a logical strategy to the MOP, the prime goal of solving the MOP is to obtain a Paretooptimal set. The Pareto-optimal solutions can be obtained by solving on a one-at-a-time basis using single objective optimization methods like weighted sum method and the $\varepsilon$ constraint method. Unlike the conventional methods, population-based methods (e.g. evolutionary algorithms) such as genetic algorithm, particle swarm optimization, simulated annealing and differential evolution can generate Paretooptimal set simultaneously. The searching strategy of different evolutionary algorithms is different and they are based imitating some natural processes. However, the common theme of all the evolutionary algorithms is to search the whole hyper-domain of decision variables and find the best possible solution. Population based methods are further subdivided into non-Pareto approaches and Pareto approaches. In non-Pareto approach, the selection/reproduction of the new population in the subsequent generations are based on the objective function values whereas in Pareto-approaches, the new population is generated not only on the basis of the objective value themselves but also on their dominance property. In this work, we used the Pareto-based approach known as nondomination based genetic algorithm (NSGA-II) which was proposed by Deb et al., (2000) because of its elitism and minimal computational complexity. Genetic algorithms (GAs) which imitate the process of natural evolution have shown successful results in many optimization problems which are difficult to solve by the conventional methods of the mathematical programming (Nemhauser et al., 1989).

4.1 Non-domination based genetic algorithm for multiobjective optimization

A brief introduction to the NSGA algorithm (Deb et al., 2000) is provided here. The algorithm is initiated with suitable values for population size and number of generations. The stopping criterion of the algorithm is the maximum number of generations. Broadly, the prime steps involved in the algorithm in each generation are selection, offspring production and recombination. First, the population is initialized randomly within the bounds of the decision variables. Once the population is initialized, they are sorted into separate fronts based on non-domination as discussed earlier. Among these fronts, the first front members completely dominate others in the current population and the second front members are dominated by only the first front members and so on. Each individual in the front is given a rank (fitness) based on the front in which they are present. The first front individuals are assigned a fitness value of 1 and second front individuals are assigned fitness value of 2 and so on. Apart from this, a parameter called crowding distance is calculated for each individual. The interpretation of the crowding distance is the closeness of an individual to its neighbours. A larger crowding distance indicates the diverse nature of the population. Crowding distance is compared only when the individuals belong to the same front. Thus, best $N$ parent individuals are selected from the current population based on the rank and crowding distance, where $N$ is the population size. Then, in the offspring production step, the selected parent individuals are used to generate offspring via the crossover and mutation operators. Finally, in the recombination step, the offspring population is combined with the current generation population and the combined population is used as an initialized population for the next generation. In this way, the procedure is repeated until the maximum number of generations. Thus, the important tuning parameters in this algorithm are the number of generations, indices for crossover and mutation processes. The schematic representation of the algorithm is shown in Fig. 2.

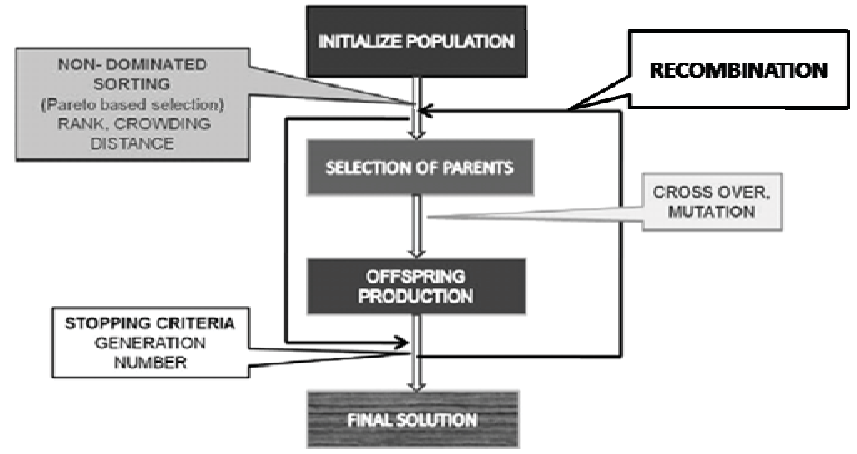

Fig.2. Non-dominated sorting genetic algorithm

\section{MATHEMATICAL MODEL}

The model taken from Piccoli and Castiglione (2006) describes the interactions among the tumor cells, helper and cytotoxic T-cells, dendritic cells and interleukin. The model assumes that tumor cells are immunogenic and do not metastasize. In other words, tumor cells are recognized by dendritic cells and are presented to cytotoxic T-cells. The interactions between the cytotoxic $\mathrm{T}$ lymphocytes and the tumor cells are described by a kinetic scheme and are presented in the form of ordinary differential equations. The states in the system are denoted by

- $H(t)$, helper T-cells (CD4+)

- $\quad C(t)$, cytotoxic T-cells (CD8+)

- $M(t)$, tumor cells

- $\quad D(t)$, dendritic cells

- $I(t)$, interleukin

The pharmacodynamics is represented by the term $e_{2} M C$ in equation (3) and the pharmacokinetics is captured in equation (4). In equation (4), ' $u$ ' is the input rate of the dendritic cells. Once the dendritic cells are injected, the CD4+ cells, CD8+ cells, and interleukins are triggered as shown in equations (1, $2 \& 5)$ respectively by the following terms. $\left(c_{0} D d_{0} \gamma\left(H, f_{0}\right)\right.$, $\left.c_{1} I(M+D) d_{1} \gamma\left(C, f_{1}\right), \quad a_{4} H D\right)$. The first two terms in equations (1) and (2) correspond to the natural evolution of 
the cytotoxic T-lymphocytes. In the same way, the second and third terms in equation (5) explain the loss of interleukin (IL-2) due to its interactions with CD8+ cells and its natural decay respectively. The model assumes that tumor growth follows the logistic equation with the constants ' $d_{2}$ ' and ' $f_{2}$ ' in the absence of immune interactions. Another assumption in the model is that externally administered DCV is the only source of dendritic cells. The initial value of tumor is $M(0)=$ 0.1 , the initial level of helper and cytotoxic T-cells are taken to be their equilibrium values $H(0)=\mathrm{a}_{0} / \mathrm{b}_{0}$ and $C(0)=\mathrm{a}_{1} / \mathrm{b}_{1}$. It is assumed that there is no immune response before the treatment and therefore $I(0)$ and $D(0)$ are taken to be zero.

\subsection{Model equations}

$\frac{d H}{d t}=a_{0}-b_{0} H+c_{0} D d_{0} \gamma\left(H, f_{0}\right)$,

$\frac{d C}{d t}=a_{1}-b_{1} C+c_{1} I(M+D) d_{1} \gamma\left(C, f_{1}\right)$,

$\frac{d M}{d t}=d_{2} \gamma\left(M, f_{2}\right)-e_{2} M C$,

$\frac{d D}{d t}=-e_{3} D C+u$,

$\frac{d I}{d t}=a_{4} H D-c_{4} C I-e_{4} I$,

where $\gamma(x, c)=x(1-x / c)$

\subsection{Problem formulation:}

Objective 1: $\min _{u\left(t_{i}\right)} \int_{0}^{t_{f}} M\left(t, u\left(t_{i}\right)\right) d t+M\left(t_{f}\right)$

Objective 2: $\min _{u\left(t_{i}\right)}\left(\operatorname{Max}\left(M\left(t, u\left(t_{i}\right)\right)\right)\right)$

$t_{f}$ is the final time i.e. the planning horizon and $t_{i}$ is the $\mathrm{i}^{\text {th }}$ time injection of the dendritic cells.

\section{Constraints:}

Equations (1) through (5) i.e. the mathematical model and $t_{i-1} \leq t_{i} \leq t_{i+1}, i=1,2 \ldots ., 9$

In this paper, two objectives are considered based on the typical goals sought by oncologists. Objective 1, as given by equation (6), seeks to minimize the summation of the running load and final load of tumor cells. Objective 2 (equation (7)) seeks to minimize the maximum possible value of the tumor cells in the given time horizon. If only objective 1 is taken into consideration, it may so happen that the tumor cells may shoot up to a very high value at a particular time while remaining at lower values at other times. This sudden shoot up of tumor cells to a higher value may lead to later stages of cancer which is very difficult to treat. So, in order to maintain the tumor in the benign stage, objective 2 is also considered. The time horizon considered to implement the multiobjective optimization is 4500 hours (approximately 6 months). In the given time horizon, the plan is to give injections of DCV ten times. Thus, the decision variables of the problem are the time of injections. It is assumed that the duration of injection every time is one hour and total vaccine quantity given in each injection is 0.5 . Thus, $u\left(t_{i}\right)$ is equal to $0.5 \mathrm{c} \mathrm{mm}^{-3} \mathrm{~h}^{-1}$.

Table 1. Parameter values

\begin{tabular}{|c|c|c|c|}
\hline Parameter & Description & Value & $\begin{array}{c}\text { Units } \\
(\mathrm{c}=\text { cells, } \\
\mathrm{h}=\text { hours })\end{array}$ \\
\hline $\mathrm{a}_{0}$ & CD4 $\mathrm{T}$ birth rate & $10^{-4}$ & $\mathrm{c} \mathrm{h}^{-1} \mathrm{~mm}^{-3}$ \\
\hline $\mathrm{b}_{0}$ & CD4 $\mathrm{T}$ death rate & 0.005 & $\mathrm{~h}^{-1}$ \\
\hline $\mathrm{c}_{0}$ & $\begin{array}{l}\text { Max. proliferation of } \\
\text { CD4 T }\end{array}$ & 10 & \\
\hline $\mathrm{d}_{0}$ & $\begin{array}{l}1 / 2 \text { saturation constant } \\
\text { of CD4 T }\end{array}$ & $10^{-2}$ & $\mathrm{c}^{-1} \mathrm{~h}^{-1} \mathrm{~mm}^{3}$ \\
\hline $\mathrm{f}_{0}$ & $\begin{array}{l}\text { Carrying capacity of } \\
\text { CD4 T }\end{array}$ & 1 & $\mathrm{c} \mathrm{mm^{-3 }}$ \\
\hline$a_{1}$ & CD8 $\mathrm{T}$ birth rate & $10^{-4}$ & $\mathrm{c} \mathrm{h}^{-1} \mathrm{~mm}^{3}$ \\
\hline $\mathrm{b}_{1}$ & CD8 $T$ death rate & 0.005 & $\mathrm{~h}^{-1}$ \\
\hline $\mathrm{c}_{1}$ & $\begin{array}{l}\text { Max. proliferation of } \\
\text { CD8 T }\end{array}$ & 10 & \\
\hline $\mathrm{d}_{1}$ & $\begin{array}{l}1 / 2 \text { saturation constant } \\
\text { of CD8 T }\end{array}$ & $10^{-2}$ & $\mathrm{~h}^{-1}\left(\mathrm{~mm}^{-3} / \mathrm{c}\right)^{2}$ \\
\hline $\mathrm{f}_{1}$ & $\begin{array}{l}\text { Carrying capacity of } \\
\text { CD8 T }\end{array}$ & 1 & $\mathrm{c} \mathrm{mm}^{-3}$ \\
\hline $\mathrm{d}_{2}$ & $\begin{array}{l}1 / 2 \text { saturation constant } \\
\text { of tumor }\end{array}$ & 0.02 & $\mathrm{~h}^{-1}$ \\
\hline$e_{2}$ & $\begin{array}{l}\text { Killing by CD8 of } \\
\text { tumor }\end{array}$ & 0.1 & $\mathrm{c}^{-1} \mathrm{~h}^{-1} \mathrm{~mm}^{3}$ \\
\hline $\mathrm{f}_{2}$ & $\begin{array}{l}\text { Carrying capacity of } \\
\text { tumor }\end{array}$ & 1 & $\mathrm{c} \mathrm{mm}^{-3}$ \\
\hline$e_{3}$ & CD8 $\mathrm{T}$ killing of DC & 0.1 & $\mathrm{c}^{-1} \mathrm{~h}^{-1} \mathrm{~mm}^{3}$ \\
\hline $\mathrm{a}_{4}$ & $\begin{array}{l}\text { IL-2 production by } \\
\text { CD4 T }\end{array}$ & $10^{-2}$ & $\mathrm{c}^{-1} \mathrm{~h}^{-1} \mathrm{~mm}^{3}$ \\
\hline $\mathrm{c}_{4}$ & $\begin{array}{l}\text { IL-2 uptake by CD } 8 \\
\mathrm{~T}\end{array}$ & $10^{-7}$ & $\mathrm{c}^{-1} \mathrm{~h}^{-1} \mathrm{~mm}^{3}$ \\
\hline $\mathrm{e}_{4}$ & IL-2 degradation rate & $10^{-2}$ & $\mathrm{~h}^{-1}$ \\
\hline
\end{tabular}




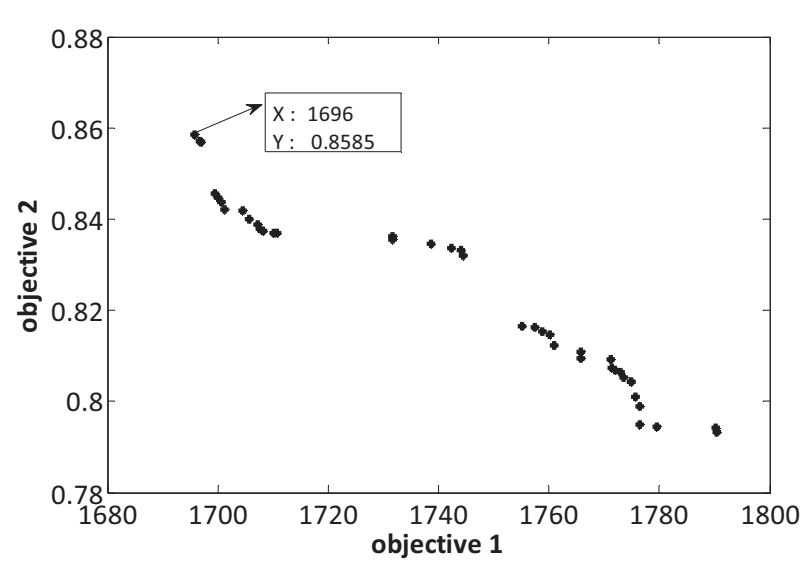

Fig.3. Optimal Pareto solutions for the multi-objective optimization problem

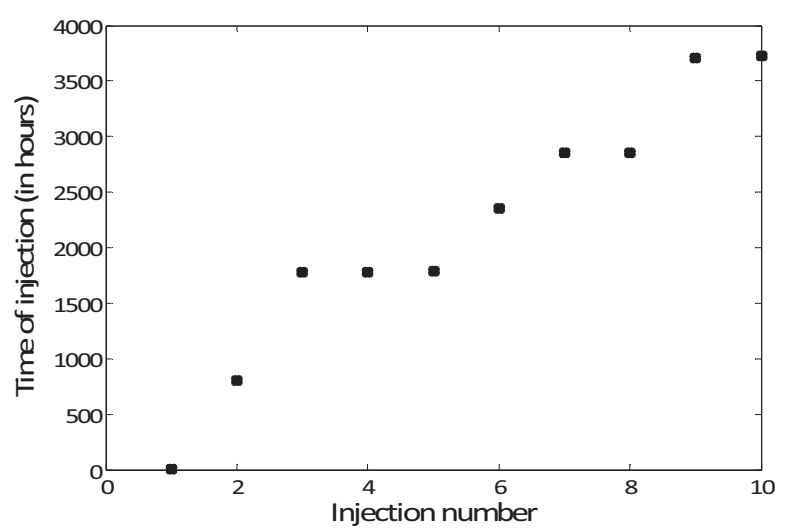

Fig.4. Plot of time of injections of the dendritic cell vaccine for the chosen solution

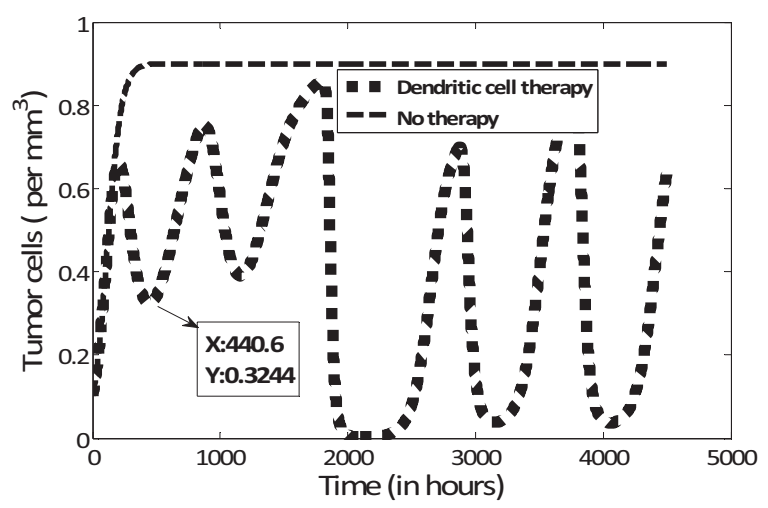

Fig.5. Dynamics of tumor cells for no therapy and dendritic cell therapy

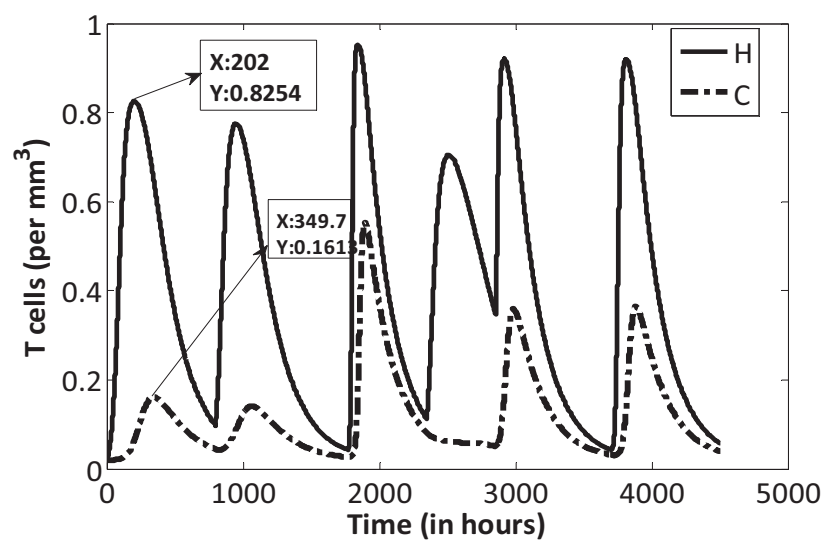

Fig.6. Dynamics of helper T-cells and cytotoxic T-cells

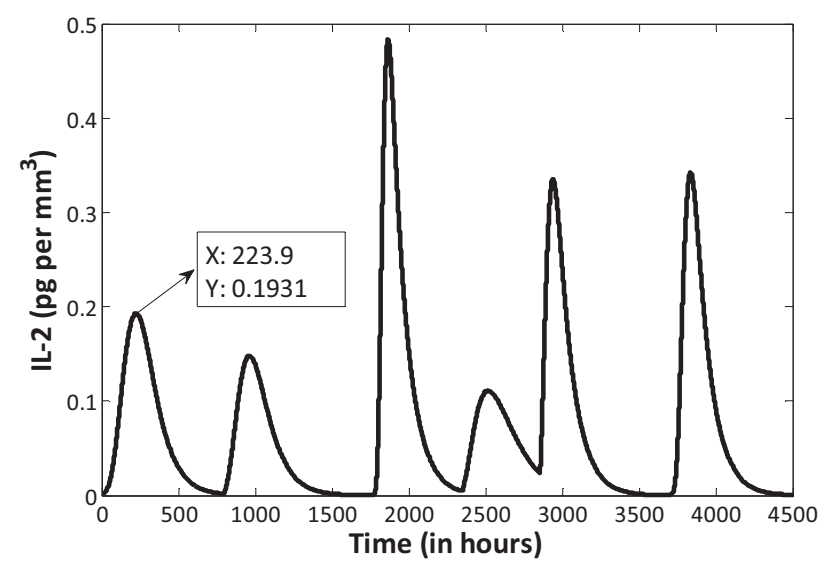

Fig.7. Dynamics of interleukin

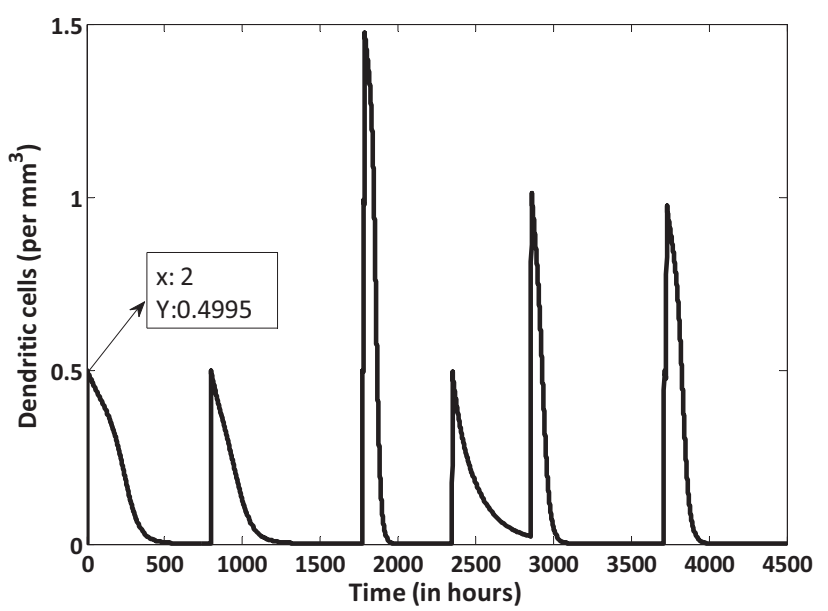

Fig.8. Dynamics of dendritic cells 


\section{RESULTS AND DISCUSSION}

We used MATLAB's implementation of NSGA-II to solve the multi-objective optimization problem as outlined above. The algorithm starts with an initial population of possible solutions within their mentioned bounds and in each generation, the solutions are updated based on the genetic principles. In this work, we considered the population number to be 40 and the maximum number of generations as 40 .

Finally, the algorithm provides the best Pareto curve as shown in Fig.3. It can be seen from the Fig.3 that there are gaps in the Pareto curve in spite of the problem being continuous. These gaps can be filled by increasing the population number and the number of generations of the genetic algorithm, but at the cost of higher computational effort. Then, one of the solutions can be chosen from the Pareto curve as per the requirement. Here, solution marked in Fig 3. is chosen because it corresponds to the least value of objective 1 (tumor burden) among all points of the Pareto curve. The time of injections for the chosen point from the Pareto curve is given in Fig 4. According to this treatment plan, DCV is injected and the evolution of the tumor cells, Tcells, interleukin, and dendritic cells are shown in figures 5 through 8 respectively.

From Fig.4, it is observed that injection timings are almost close during (1700-1800 hours, 2850-2900 hours, and 37003750 hours). The reason is the increase of tumor cells during these periods. So, in order to bring them down immediately, the frequency of injection times is increased. Thus, the treatment planning seems to be reasonable and logical.

From figures 5 through 8 , the first intervention of dendritic cells is considered. This intervention is given in the second hour. Because of this intervention, the helper T-cells, interleukin, and cytotoxic T-cells reach their highest value at around 200 hours ( 8 days), 221.9 hours (9 days), 349.7 hours (15 days) respectively from the time of intervention of DCV. At the same time, tumor cells reach a lower value at around 440.6 hours (19 days) due to the first intervention. Similarly, tumor cells took around 15 days to reach its lower value after second intervention of DCV. Thus, for the given input of $\mathrm{DCV}$, the time gap between the interventions should not be more than 3 weeks in order to avoid the peak value of the tumor cells. Moreover, it takes more time for the helper Tcells and cytotoxic T-cells to get activated before reacting to the tumor cells. This activation time can be reduced by injecting more DCV (but within the threshold range) or by introducing the interleukin therapy (approved by FDA) where interleukin is also given externally. One of our future plans is to consider a combination of dendritic cell therapy and interleukin therapy.

\section{CONCLUSIONS}

We have applied multi-objective optimization to find the optimal schedule of dendritic cell therapy for a given input of dendritic cells. The freedom of choice of any one solution as per the requirements is a major advantage of multi-objective optimization strategy. The obtained treatment planning seems to be reasonable in controlling the tumor cells from reaching a higher value. Further analysis suggests that the time gap between the interventions should be less than 3 weeks in order to achieve the objective. Thus, the obtained protocol design can guide caregivers in treating cancer subjects.

\section{ACKNOWLEDGEMENTS}

This work was supported by the National University of Singapore in the form of a research scholarship to the first author.

\section{REFERENCES}

Adam, J.K., Odhav, B. and Bhoola, K.D. (2003) Immune responses in cancer, Pharmacology \& Therapeutics, 99, 113132.

Cappuccio, A., Castiglione, F. and Piccoli, B. (2007) Determination of the optimal therapeutic protocols in cancer immunotherapy, Mathematical Biosciences, 209, 1-13.

Castiglione, F. and Piccoli, B. (2007) Cancer immunotherapy, mathematical modeling and optimal control, Journal of Theoretical Biology, 247, 723-732.

De Boer, R.J. and Hogeweg, P. (1986) Interactions between macrophages and T-lymphocytes: Tumor sneaking through intrinsic to helper $\mathrm{T}$ cell dynamics, Journal of Theoretical Biology, 120, 331-351.

de Pillis, L.G., Gu, W. and Radunskaya, A.E. (2006) Mixed immunotherapy and chemotherapy of tumors: modeling, applications and biological interpretations, Journal of Theoretical Biology, 238, 841-862.

de Pillis, L.G., Radunskaya, A.E. and Wiseman, C.L. (2005) A Validated Mathematical Model of Cell-Mediated Immune Response to Tumor Growth, Cancer Res, 65, 7950-7958.

Deb, K., Agrawal, S., Pratap, A. and Meyarivan, T. (2000) A fast elitist non-dominated sorting genetic algorithm for multiobjective optimization: NSGA-II. Springer-Verlag, Paris, France, 849-858.

Dua, P., Dua, V. and Pistikopoulos, E.N. (2008) Optimal delivery of chemotherapeutic agents in cancer, Computers \& Chemical Engineering, 32, 99-107.

Hanahan, D. and Weinberg, R.A. (2000) The Hallmarks of Cancer, Cell, 100, 57-70.

Kirschner, D. and Panetta, J.C. (1998) Modeling immunotherapy of the tumor - immune interaction, Journal of Mathematical Biology, 37, 235-252.

Kuznetsov, V.A., Makalkin, I.A., Taylor, M.A. and Perelson, A.S. (1994) Nonlinear dynamics of immunogenic tumors: Parameter estimation and global bifurcation analysis, Bulletin of Mathematical Biology, 56, 295-321. 
Martins, M.L., Ferreira Jr, S.C. and Vilela, M.J. (2007) Multiscale models for the growth of avascular tumors, Physics of Life Reviews, 4, 128-156.

Nemhauser, G.L., Rinnooy Kan, A.H.G. and Todd, M.J. (1989) Optimization. North-Holland.

Paola Pisani, F.B., D. Maxwell Parkin, (2002) Estimates of the world-wide prevalence of cancer for 25 sites in the adult population, International Journal of Cancer, 97, 72-81.

Piccoli, B. and Castiglione, F. (2006) Optimal vaccine scheduling in cancer immunotherapy, Physica a-Statistical Mechanics and Its Applications, 370, 672-680.

Szymanska, Z. (2003) Analysis of immunotherapy models in the context of cancer dynamics, Int.J. Appl. Math. Comput. Sci., 13, 407- 418.

Tamaki, H., Kita, H. and Kobayashi, S. (1996) Multiobjective optimization by genetic algorithms: a review. Proceedings of IEEE International Conference on Evolutionary Computation. 517-522. 